\title{
THERMOELASTIC WAVE IN METAL INDUCED BY ULTRAFAST LASER PULSES
}

\author{
Xinwei Wang and Xianfan Xu \\ School of Mechanical Engineering \\ Purdue University \\ West Lafayette, Indiana, USA
}

\begin{abstract}
In this work, an analytical solution for thermoelastic waves in a metal induced by an ultrafast laser pulse was formulated in the form of a Fourier series. The two-step heat transfer model was applied to account for the thermal nonequilibrium between electrons and the lattice during ultrafast pulsed laser heating. The coupling between the strain rate and the temperature field was also considered. Calculations were carried out to study generation, propagation, and attenuation of thermoelastic waves induced by a pico- and a femtosecond laser pulse. Results were compared with those obtained without considering the two-step heat transfer effect.
\end{abstract}

In recent years, ultrafast pulsed lasers (lasers with a pulse width of the order of picoseconds or less) are being rapidly developed. Many applications using ultrafast lasers are being investigated, including micromachining (e.g., [1-5]), nondestructive detection [6], and materials characterization [7-9]. Thermal phenomena induced by ultrafast laser heating are different from those induced by longer laser pulses. Due to the extremely short heating time, modifications to the Fourier heat conduction theory are necessary in order to predict the temperature field correctly. One modification is to consider the non-Fourier effect, which accounts for a thermal relaxation time in the energy carrier's collision process. At low temperatures, the non-Fourier effect could cause a wave behavior of heat transfer [10-13]. The other modification is to employ the two-temperature (or two-step heat transfer) model [14] to account for the fact that the photon energy is first absorbed by the electron gas in a metal and is then transferred to the lattice. Using this model, the temperature of the electron gas was found to be much higher than that of the lattice during the initial period of ultrafast laser heating [15-17].

During pulsed laser heating, a thermoelastic wave is generated due to thermal expansion in the near surface region and propagates into the target. Because of the extremely short heating time, the laser-induced thermoelastic wave has an extremely

Received 28 March 2001; accepted 27 September 2001.

Support by the National Science Foundation (CTS-9624890) is gratefully acknowledged.

Address correspondence to Professor Xianfan Xu, School of Mechanical Engineering, Purdue University, West Lafayette, IN 47907. Email: xxu@ecn.purdue.edu 
high strain rate, which in turn causes strong coupling between the strain rate and the temperature field. This coupling damps the stress wave during its propagation and induces a localized temperature variation [13].

Thermoelastic waves induced by laser heating have been studied extensively. Commonly, one seeks analytical solutions owing to the insufficient resolution of numerical techniques. A large amount of work has been conducted to solve thermoelastic wave problems without including the non-Fourier effect or the two-step heat transfer effect [18-29], while some recent investigations considered the nonFourier effect by introducing a single relaxation time or a dual phase-lag in the coupled heat transfer and displacement equations [17, 30, 31]. Using Fourier series, Wang and $\mathrm{Xu}$ developed an analytical solution to heat transfer and thermoelastic waves in dielectrics with the consideration of the non-Fourier effect [13]. It was found that the non-Fourier effect caused a higher surface temperature increase, a weaker stress wave, and a second stress wave propagating at the same speed as the thermal wave. Also, attenuation of the stress wave and expansion of its duration were predicted as a result of the coupling between the strain rate and the temperature field.

In this article, the previous solution for a dielectric material [13] is extended to a metal, and the thermal nonequilibrium between electrons and the lattice in lasermetal interaction is considered. The solution is formulated in the form of a Fourier series. The two-step heat transfer and the coupling between the strain rate and the lattice temperature field are also taken into account. The temperature field development and stress generation and attenuation are studied. In order to illustrate the two-step heat transfer effect, the results are also compared with those obtained without considering the two-step heat transfer.

\section{THEORETICAL ANALYSIS}

In this section, solutions to the thermoelastic wave in a metal induced by an ultrafast pulsed laser are derived. First, the laser pulse energy is expressed as a Fourier series. Thermoelastic waves induced by each laser energy term in the Fourier series are derived and then summed to represent the thermoelastic wave induced by a laser pulse. The laser fluence is chosen to raise the lattice temperature close to the melting point in order to investigate the maximum possible stress before phase change occurs.

It is assumed that the repetition rate of the laser pulses is low. Therefore, the influence from the preceding pulses can be neglected, and the solution of the temperature and stress wave reflects those of a single pulse [32-36]. A homogeneous halfspace metal is irradiated by a pulsed laser. The coordinate $x$ is chosen to originate from the surface and point toward the inside of the target. For the one-dimensional problem, the governing equations for the temperatures of electrons and the lattice, $T_{\mathrm{e}}$ and $T_{1}$, and the lattice displacement $u$, consist of three coupled partial differential equations $[20,37]$

$$
C_{\mathrm{e}} \frac{\partial T_{\mathrm{e}}}{\partial t}=\frac{\partial}{\partial x}\left(k \frac{\partial T_{\mathrm{e}}}{\partial x}\right)-G_{\mathrm{el}}\left(T_{\mathrm{e}}-T_{\mathrm{l}}\right)+\beta I(t) e^{-\beta x}
$$




$$
\begin{gathered}
C_{1} \frac{\partial T_{1}}{\partial t}=G_{\mathrm{el}}\left(T_{\mathrm{e}}-T_{1}\right)-B \beta_{\mathrm{T}} T_{0} \frac{\partial^{2} u}{\partial x \partial t} \\
\rho \frac{\partial^{2} u}{\partial t^{2}}=\left(B+\frac{4}{3} G\right) \frac{\partial^{2} u}{\partial x^{2}}-B \beta_{\mathrm{T}} \frac{\partial T_{1}}{\partial x}
\end{gathered}
$$

In these equations, $C_{\mathrm{e}}$ and $C_{1}$ denote the volumetric heat capacity of electrons and the lattice, $k$ is the thermal conductivity of the electron gas, $\rho$ is the density, $\beta$ is the optical absorption coefficient, $\beta_{\mathrm{T}}$ is the volumetric thermal expansion coefficient, $B$ and $G$ are the bulk and shear moduli, and $T_{0}$ is the initial temperature of the target.

Equation (1a) describes the electron temperature increase due to absorption of the photon energy $(\beta I \exp (-\beta x))$, heat diffusion $\left(\partial\left(k \partial T_{\mathrm{e}} / \partial x\right) / \partial x\right)$, and transfer of the electron energy to the lattice $\left(G_{\mathrm{el}}\left(T_{\mathrm{e}}-T_{1}\right)\right)$, which is proportional to the temperature difference between electrons and the lattice. Equation $(1 b)$ governs the lattice temperature increase due to energy transfer from the electron gas $\left(G_{\mathrm{el}}\left(T_{\mathrm{e}}-T_{1}\right)\right)$ and the coupling between the temperature field and the strain rate, $B \beta_{\mathrm{T}} T_{0} \partial^{2} u /(\partial x \partial t)$. Heat diffusion is not considered in Eq. (1b) since heat is conducted much more rapidly through electrons than the lattice [37]. Equation $(1 c)$ is the equation of displacement, which accounts for thermal expansion. Generation and propagation of the elastic wave is due to lattice thermal expansion and vibration; therefore only the lattice temperature appears in the displacement equation.

The coupling factor between electrons and the lattice, $G_{\mathrm{el}}$, is calculated as [17]

$$
G_{\mathrm{el}}=\frac{\pi^{4}\left(n_{\mathrm{e}} v_{\mathrm{s}} k_{\mathrm{B}}\right)^{2}}{18 k}
$$

where $n_{\mathrm{e}}$ is the density of free electrons per unit volume, $v_{\mathrm{S}}$ is the speed of sound, and $k_{\mathrm{B}}$ is the Boltzmann constant. In this calculation, the value of $G_{\mathrm{el}}$ is obtained from [17]. The ratio $C_{\mathrm{e}} / G_{\mathrm{el}}$ represents a time constant for the electron-lattice interaction. For gold, this time constant is $0.75 \mathrm{ps}$ [17].

Thermophysical properties of electrons and the lattice are temperature dependent. For instance, the specific heat, the thermal conductivity of electrons, and the coupling factor between electrons and the lattice increase with the electron temperature [38, 39]. However, it is not feasible to account for temperature dependency of the properties in developing analytical solutions. In this work, constant properties are used, which will affect the accuracy of the results. With this in mind, interpretation of the results is focused more on the phenomena involved rather than absolute numerical values.

By introducing $\theta_{\mathrm{e}}=T_{\mathrm{e}}-T_{0}, \quad \theta_{\mathrm{l}}=T_{1}-T_{0}, \quad c_{\mathrm{e}}=\sqrt{(B+4 / 3 \cdot G) / \rho}, \quad$ and $v=-B \beta_{\mathrm{T}} /(B+4 / 3 \cdot G)$, Eqs. $(1 a-1 c)$ are rewritten as

$$
\begin{gathered}
C_{\mathrm{e}} \frac{\partial \theta_{\mathrm{e}}}{\partial t}=\frac{\partial}{\partial x}\left(k \frac{\partial \theta_{\mathrm{e}}}{\partial x}\right)-G_{\mathrm{el}}\left(\theta_{\mathrm{e}}-\theta_{1}\right)+\beta I e^{-\beta x} \\
C_{1} \frac{\partial \theta_{1}}{\partial t}=G_{\mathrm{el}}\left(\theta_{\mathrm{e}}-\theta_{1}\right)-B \beta_{\mathrm{T}} T_{0} \frac{\partial^{2} u}{\partial x \partial t}
\end{gathered}
$$




$$
\frac{1}{c_{\mathrm{e}}^{2}} \frac{\partial^{2} u}{\partial t^{2}}=\frac{\partial^{2} u}{\partial x^{2}}+v \frac{\partial \theta_{1}}{\partial x}
$$

Before heating, the sample is assumed to have a uniform temperature, no displacement, and stress-free and the first-order derivatives of temperature and displacement to time are taken as zero. The surface of the sample is assumed to be thermally insulated and stress-free. At $x \rightarrow \infty$, the sample is fixed and has no temperature increase and stress. These initial and boundary conditions are expressed as

$$
\begin{gathered}
\theta_{\mathrm{e}}=0 \quad \text { at } t=0, x \geq 0 \\
\theta_{1}=0 \quad \text { at } t=0, x \geq 0 \\
u=0 \quad \text { at } t=0, x \geq 0 \\
\frac{\partial \theta_{\mathrm{e}}}{\partial t}=0 \quad \text { at } t=0, x \geq 0 \\
\frac{\partial \theta_{1}}{\partial t}=0 \quad \text { at } t=0, x \geq 0 \\
\frac{\partial u}{\partial t}=0 \quad \text { at } t=0, x \geq 0 \\
\frac{\partial \theta_{\mathrm{e}}}{\partial x}=0 \quad \text { at } x=0, t>0 \\
\frac{\partial \theta_{1}}{\partial x}=0 \quad \text { at } x=0, t>0 \\
\frac{\partial u}{\partial x}+v \theta_{1}=0 \quad \text { at } x=0, t>0
\end{gathered}
$$

As mentioned previously, the first step to solving Eqs. (1-3) is to represent the laser pulse intensity in terms of a Fourier series

$$
I(t)=a_{0}+\sum_{i=1}^{\infty}\left(a_{i} \cos (\omega t)+b_{i} \sin (\omega t)\right)
$$

where $a_{\mathrm{i}}$ and $b_{\mathrm{i}}$ are coefficients of Fourier series, $\omega=i \omega_{0}$, and $\omega_{0}=2 \pi f_{0}$ where $f_{0}$ is the repetition rate of the laser pulses. The temperature increase induced by $I(t)$ consists of two parts. One is the steady temperature increase, $\bar{\theta}_{\mathrm{e}}$ and $\bar{\theta}_{1}$, caused by $a_{0}$ in Eq. (4); and the other one is the transient part, $\tilde{\theta}_{\mathrm{e}}$ and $\tilde{\theta}_{1}$, caused by all other terms in Eq. (4). In the solution, the steady temperature increase is to be maintained at zero at the beginning of each laser pulse.

Because of the linear relations among the temporal parts $\tilde{\theta}_{\mathrm{e}}, \tilde{\theta}_{1}, \tilde{u}$, and $I(t)$, as long as the laser beam intensity is expressed with Eq. (4), $\tilde{\theta}_{\mathrm{e}}, \tilde{\theta}_{1}, \tilde{u}$, and the stress $\sigma$ can be written as

$$
\tilde{\theta}_{\mathrm{e}}=\sum_{i=1}^{\infty}\left(a_{i} \operatorname{Re}\left(\tilde{\theta}_{\mathrm{e}, i}\right)+b_{i} \operatorname{Im}\left(\tilde{\theta}_{\mathrm{e}, i}\right)\right)
$$




$$
\begin{aligned}
\tilde{\theta}_{1} & =\sum_{i=1}^{\infty}\left(a_{i} \operatorname{Re}\left(\tilde{\theta}_{1, i}\right)+b_{i} \operatorname{Im}\left(\tilde{\theta}_{1, i}\right)\right) \\
\tilde{u} & =\sum_{i=1}^{\infty}\left(a_{i} \operatorname{Re}\left(\tilde{u}_{i}\right)+b_{i} \operatorname{Im}\left(\tilde{u}_{i}\right)\right) \\
\sigma & =\sum_{i=1}^{\infty}\left(a_{i} \operatorname{Re}\left(\tilde{\sigma}_{i}\right)+b_{i} \operatorname{Im}\left(\tilde{\sigma}_{i}\right)\right)
\end{aligned}
$$

where $\tilde{\theta}_{\mathrm{e}, i}, \tilde{\theta}_{1, i}, \tilde{u}_{i}$, and $\tilde{\sigma}_{i}$ are the temperature increase of electrons and the lattice, the lattice displacement, and the stress induced by a laser beam with a complex intensity of $\exp (j \omega t)$. Re and Im denote the real and imaginary parts of a complex number. $\tilde{\theta}_{\mathrm{e}, i}, \tilde{\theta}_{1, i}, \tilde{u}_{i}$, and $\tilde{\sigma}_{i}$ should vary with time in the same manner as the complex laser intensity does; therefore, they have the form of $\tilde{\theta}_{\mathrm{e}, i}=\theta_{\mathrm{e}, i} e^{j \omega t}, \tilde{\theta}_{1, i}=\theta_{1, i} e^{j \omega t}$, $\tilde{u}_{i}=u_{i} e^{j \omega t}$, and $\tilde{\sigma}_{i}=\sigma_{i} e^{j \omega t}$.

According to Eq. (2b), the electron temperature can be expressed in terms of the lattice temperature, its time derivative, and the strain rate. Thus, combining Eqs. (2a) and $(2 b)$ would eliminate the electron temperature from Eq. (2a). By introducing $\psi_{1}=j \omega \cdot\left(C_{1}+C_{\mathrm{e}}\right) / k-C_{\mathrm{e}} C_{1} \omega^{2} /\left(k G_{\mathrm{el}}\right), \quad \psi_{2}=1+j \omega C_{1} / G_{\mathrm{el}}, \quad \psi_{3}=B \beta_{\mathrm{T}} T_{0} / G_{\mathrm{el}}$. $\left(C_{\mathrm{e}} \omega^{2} / k-j \omega G_{\mathrm{el}} / k\right), \psi_{4}=j \omega B \beta_{\mathrm{T}} T_{0} / G_{\mathrm{el}}$, and $\gamma=\beta / k$, the governing equations for $\theta_{1, i}, \theta_{\mathrm{e}, i}$, and $u_{i}$ are rewritten as

$$
\begin{gathered}
\psi_{1} \theta_{1, i}=\psi_{2} \frac{\partial^{2} \theta_{1, i}}{\partial x^{2}}+\psi_{3} \frac{\partial u_{i}}{\partial x}+\psi_{4} \frac{\partial^{3} u_{i}}{\partial x^{3}}+\gamma e^{-\beta x} \\
\theta_{\mathrm{e}, i}=\frac{1}{G_{\mathrm{el}}}\left(\left(j \omega C_{1}+G_{\mathrm{el}}\right) \theta_{1, i}+j \omega B \beta_{\mathrm{T}} T_{0} \frac{\partial u_{i}}{\partial x}\right) \\
-\frac{\omega^{2} u_{i}}{c_{\mathrm{e}}^{2}}=\frac{\partial^{2} u_{i}}{\partial x^{2}}+v \frac{\partial \theta_{1, i}}{\partial x}
\end{gathered}
$$

The boundary conditions for Eqs. (6) are the same as Eqs. $(3 g-3 i)$. Equations $(6 a)$ and $(6 c)$ are solved together since they are decoupled from $\theta_{\mathrm{e}, i}$. Once $\theta_{1, i}$ and $u_{i}$ become available, $\theta_{\mathrm{e}, i}$ can be solved from Eq. $(6 b)$. Due to the inhomogeneous term $\gamma \exp (-\beta x)$ in Eq. $(6 a)$, Eqs. $(6 a)$ and $(6 c)$ have particular solutions in the form of $\theta_{\mathrm{lp}, i}=A_{\mathrm{lp}, i} \exp (-\beta x)$ and $u_{\mathrm{p}, i}=B_{\mathrm{p}, i} \exp (-\beta x)$, with $A_{\mathrm{lp}, i}$ and $B_{\mathrm{p}, i}$ solved as

$$
\begin{aligned}
& A_{\mathrm{lp}, i}=\frac{\left(\beta^{2}+\omega^{2} / c_{\mathrm{e}}^{2}\right) \cdot \gamma}{\left(\psi_{1}-\psi_{2} \beta^{2}\right) \cdot\left(\beta^{2}+\omega^{2} / c_{\mathrm{e}}^{2}\right)+v \beta\left(\psi_{3} \beta+\psi_{4} \beta^{3}\right)} \\
& B_{\mathrm{p}, i}=\frac{\gamma}{\left(\psi_{1}-\psi_{2} \beta^{2}\right) \cdot\left(\beta^{2}+\omega^{2} / c_{\mathrm{e}}^{2}\right) / v \beta+\psi_{3} \beta+\psi_{4} \beta^{3}}
\end{aligned}
$$

In addition, Eqs. $(6 a)$ and $(6 c)$ have general homogeneous solutions in the form of $\theta_{\lg , i}=A_{1, i} \exp \left(k_{i} x\right)$ and $u_{\mathrm{g}, i}=B_{i} \exp \left(k_{i} x\right)$, with $A_{1, i}$, and $B_{i}$ solved from the boundary conditions. The final solution can be expressed as 


$$
\begin{gathered}
\theta_{1, i}=A_{1,1, i} e^{k_{1, i} x}+A_{1,2, i} e^{k_{2, i} x}+A_{1 \mathrm{p}, i} e^{-\beta x} \\
u_{i}=-\frac{v k_{1, i} A_{1,1, i}}{\omega^{2} / c_{\mathrm{e}}^{2}+k_{1, i}^{2}} e^{k_{1, i} x}-\frac{v k_{2, i} A_{1,2, i}}{\omega^{2} / c_{\mathrm{e}}^{2}+k_{2, i}^{2}} e^{k_{2, i} x}+B_{\mathrm{p}, i} e^{-\beta x}
\end{gathered}
$$

where

$$
\begin{gathered}
k_{i}^{2}=\frac{\psi_{1}+\psi_{3} v-\psi_{2} \omega^{2} / c_{\mathrm{e}}^{2} \pm \sqrt{\left(\psi_{1}+\psi_{3} v-\psi_{2} \omega^{2} / c_{\mathrm{e}}^{2}\right)^{2}+4\left(\psi_{2}-\psi_{4} v\right) \psi_{1} \omega^{2} / c_{\mathrm{e}}^{2}}}{2\left(\psi_{2}-\psi_{4} v\right)} \\
A_{1,1, i}=\frac{\beta A_{1 \mathrm{p}, i} p_{2, i}-p_{3, i} k_{2, i}}{k_{1, i} p_{2, i}-k_{2, i} p_{1, i}} \\
A_{1,2, i}=\frac{k_{1, i} p_{3, i}-p_{1, i} \beta A_{1 \mathrm{p}, i}}{k_{1, i} p_{2, i}-k_{2, i} p_{1, i}}
\end{gathered}
$$

In these expressions, $p_{j, i}=-v k_{j, i}^{2} /\left(\omega^{2} / c_{\mathrm{e}}^{2}+k_{j, i}^{2}\right)+v$ and $p_{3, i}=B_{\mathrm{p}, i} \beta-v A_{\mathrm{l}, i}$ with $j=1,2$. Similar to the form of $\theta_{1, i}, \theta_{\mathrm{e}, i}$ can be expressed as

$$
\theta_{\mathrm{e}, i}=A_{\mathrm{e}, 1, i} e^{k_{1, i} x}+A_{\mathrm{e}, 2, i} e^{k_{2, i} x}+A_{\mathrm{ep}, i} e^{-\beta x}
$$

where

$$
\begin{gathered}
A_{\mathrm{e}, 1, i}=\psi_{2} A_{1,1, i}+k_{1, i} \psi_{4} B_{1, i} \\
A_{\mathrm{e}, 2, i}=\psi_{2} A_{1,2, i}+k_{2, i} \psi_{4} B_{2, i} \\
A_{\mathrm{ep}, i}=\psi_{2} A_{\mathrm{lp}, i}-\beta \psi_{4} B_{\mathrm{p}, i}
\end{gathered}
$$

Using the preceding results, the stress induced by a laser intensity of $\exp (j \omega t)$ is expressed as

$$
\begin{aligned}
\sigma_{i}=\left(B+\frac{4}{3} G\right) & \cdot\left(e^{k_{1, i} x}\left(B_{1, i} k_{1, i}+v A_{1,1, i}\right)+e^{k_{2, i} x}\left(B_{2, i} k_{1, i}+v A_{1,2, i}\right)\right. \\
& \left.+e^{-\beta x}\left(-\beta B_{\mathrm{p}, i}+v A_{1 \mathrm{p}, i}\right)\right)
\end{aligned}
$$

$\tilde{\theta}_{\mathrm{e}, i}, \tilde{\theta}_{1, i}, \tilde{u}_{i}$, and $\tilde{\sigma}_{i}$ can be obtained by multiplying $\theta_{\mathrm{e}, i}, \theta_{1, i}, u_{i}$, and $\sigma_{i}$ with $\exp (j \omega t)$. The final solutions can be obtained by substituting $\tilde{\theta}_{\mathrm{e}, i}, \tilde{\theta}_{1, i}, \tilde{u}_{i}$, and $\tilde{\sigma}_{i}$ into Eqs. $(5 a-5 d)$.

\section{RESULTS AND DISCUSSION}

Using the preceding solutions, calculations were conducted to investigate the temperature fields and thermoelastic waves induced by a pico- and a femtosecond laser pulse. The influence of the two-step heat transfer on thermoelastic waves as well as 
the attenuation of thermoelastic waves due to the coupling between the strain rate and the temperature field were studied.

\section{Gold Irradiated with a Picosecond Pulsed Laser}

In this problem, a gold target is subjected to picosecond pulsed laser heating. The picosecond laser is assumed to have a base pulse width of $50 \mathrm{ps}$ and a peak intensity of $1.342 \times 10^{13} \mathrm{~W} / \mathrm{m}^{2}$ in the middle of the pulse. The peak laser intensity is chosen to raise the surface temperature close to the melting point of gold, $1337 \mathrm{~K}$. The wavelength of the laser is taken as $0.8 \mu \mathrm{m}$, which is the wavelength of the Ti:sapphire picoand femtosecond laser. The optical absorption coefficient of gold at $0.8 \mu \mathrm{m}$ is $7.51 \times 10^{7} \mathrm{~m}^{-1}$. The properties of gold used in the calculation are listed in Table 1 $[17,42,43]$.

In order to study the effect of two-step heat transfer, thermoelastic waves without considering two-step heat transfer were also calculated based on existing solutions [13].

Shown in Figure 1 are the temperature increase and the displacement at the surface with and without the two-step heat transfer model. When two-step heat transfer is considered, a large difference between the electron and lattice temperatures is observed. The electron temperature increase reaches $4200 \mathrm{~K}$ at $25 \mathrm{ps}$, while the maximum lattice temperature increase is only about $1000 \mathrm{~K}$ at $50 \mathrm{ps}$. Therefore, even though the laser pulse width $(50 \mathrm{ps})$ is much longer than the characteristic time of electron-lattice coupling $(0.75 \mathrm{ps})$, there is still a noticeable difference in their temperatures. From Figures $1 a, b$ it is seen that when two-step heat transfer is not considered, the lattice temperature increase is overpredicted and reaches a higher peak value $(1700 \mathrm{~K})$ within a shorter time. The reason for this is that a portion of energy is conducted away by the electron gas before electrons and the lattice reach thermal equilibrium, thereby resulting in the lower lattice temperature increase. Also, it takes some time to transfer energy from electrons to the lattice. For the displacement, it is seen that even though the peak displacements are the same in the two cases, the two-step heat transfer model predicts a slower displacement variation with time, which is caused by delayed heating. One interesting phenomenon is that the variation of the electron temperature closely follows the temporal shape of the laser

\section{Table 1 Properties of gold used in the calculation}

\begin{tabular}{lr}
\hline$\rho$, Density $\left(\mathrm{kg} / \mathrm{m}^{3}\right)$ & $1.93 \times 10^{4}$ \\
$k$, Thermal conductivity $(\mathrm{W} / \mathrm{m} \cdot \mathrm{K})$ & $3.15 \times 10^{2}$ \\
$C_{\mathrm{e}}$, Volumetric heat capacity of the electron gas $\left(\mathrm{J} / \mathrm{m}^{3} \cdot \mathrm{K}\right)$ & $2.1 \times 10^{4}$ \\
$C_{\mathrm{l}}$, Volumetric heat capacity of the lattice $\left(\mathrm{J} / \mathrm{m}^{3} \cdot \mathrm{K}\right)$ & $2.5 \times 10^{6}$ \\
$\beta$, Optical absorption coefficient $(1 / \mathrm{m})$ & $7.51 \times 10^{7}$ \\
$G$, Shear modulus $\left(\mathrm{kg} / \mathrm{s}^{2} \cdot \mathrm{m}\right)$ & $2.85 \times 10^{10}$ \\
$B$, Bulk modulus $\left(\mathrm{kg} / \mathrm{s}^{2} \cdot \mathrm{m}\right)$ & $1.69 \times 10^{11}$ \\
$\beta_{\mathrm{T}}$, Volumetric thermal expansion coefficient $(1 / \mathrm{K})$ & $1.42 \times 10^{-5}$ \\
$G_{\text {el }}$, Lattice-electron coupling coefficient $\left(\mathrm{W} / \mathrm{m}^{3} \cdot \mathrm{K}\right)$ & $2.8 \times 10^{16}$ \\
\hline
\end{tabular}



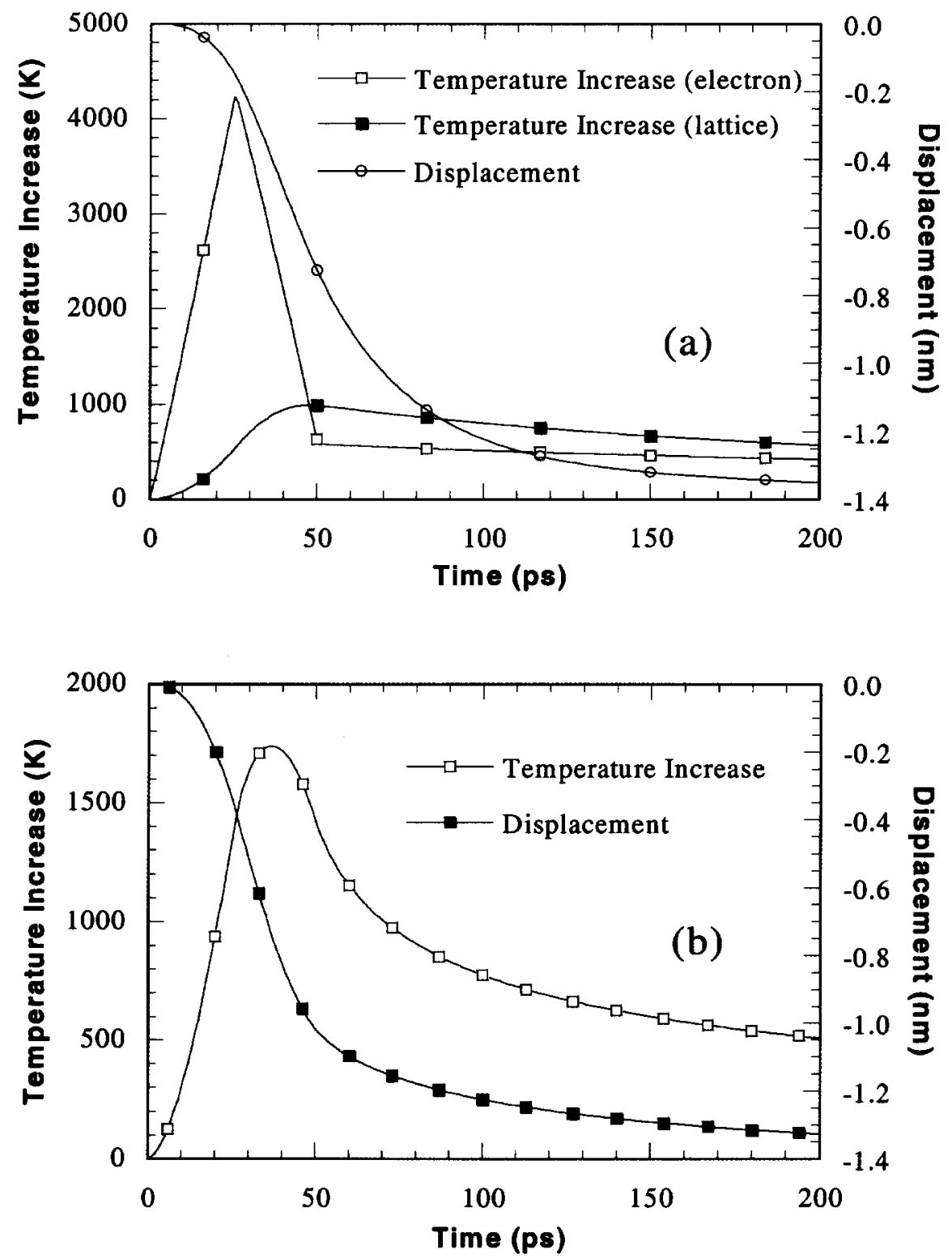

Figure 1. Temperature increase and displacement at the surface of gold illuminated with a picosecond laser $(a)$ with the consideration of two-step heat transfer model and $(b)$ without the consideration of twostep heat transfer model.

pulse. This result is verified by a numerical calculation (finite difference), which shows that the difference between the results of the two calculations is less than $2 \%$.

Figure $2 a$ shows the stress evolution when two-step heat transfer is considered. A compressive stress appears first, followed by a tensile stress. Comparing Figure $2 a$ 

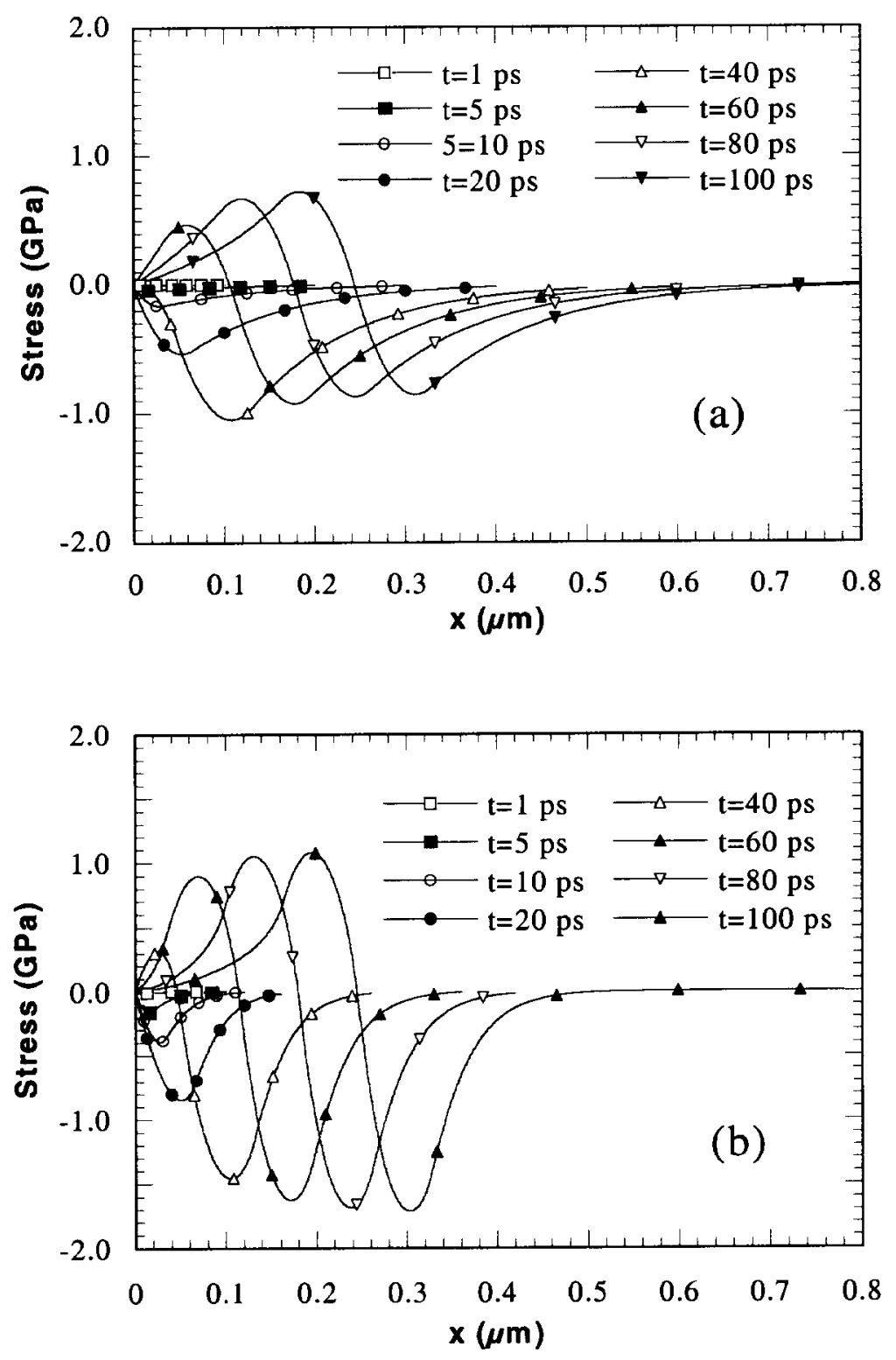

Figure 2. Development of stress waves in gold illuminated with a picosecond laser $(a)$ with the consideration of two-step heat transfer and $(b)$ without the consideration of two-step heat transfer.

with $2 b$, it is seen that the amplitude of the thermoelastic waves calculated with the two-step heat transfer model is lower than that without considering two-step heat transfer. This is due to the lower peak surface temperature and slower displacement variation when two-step heat transfer is considered. 

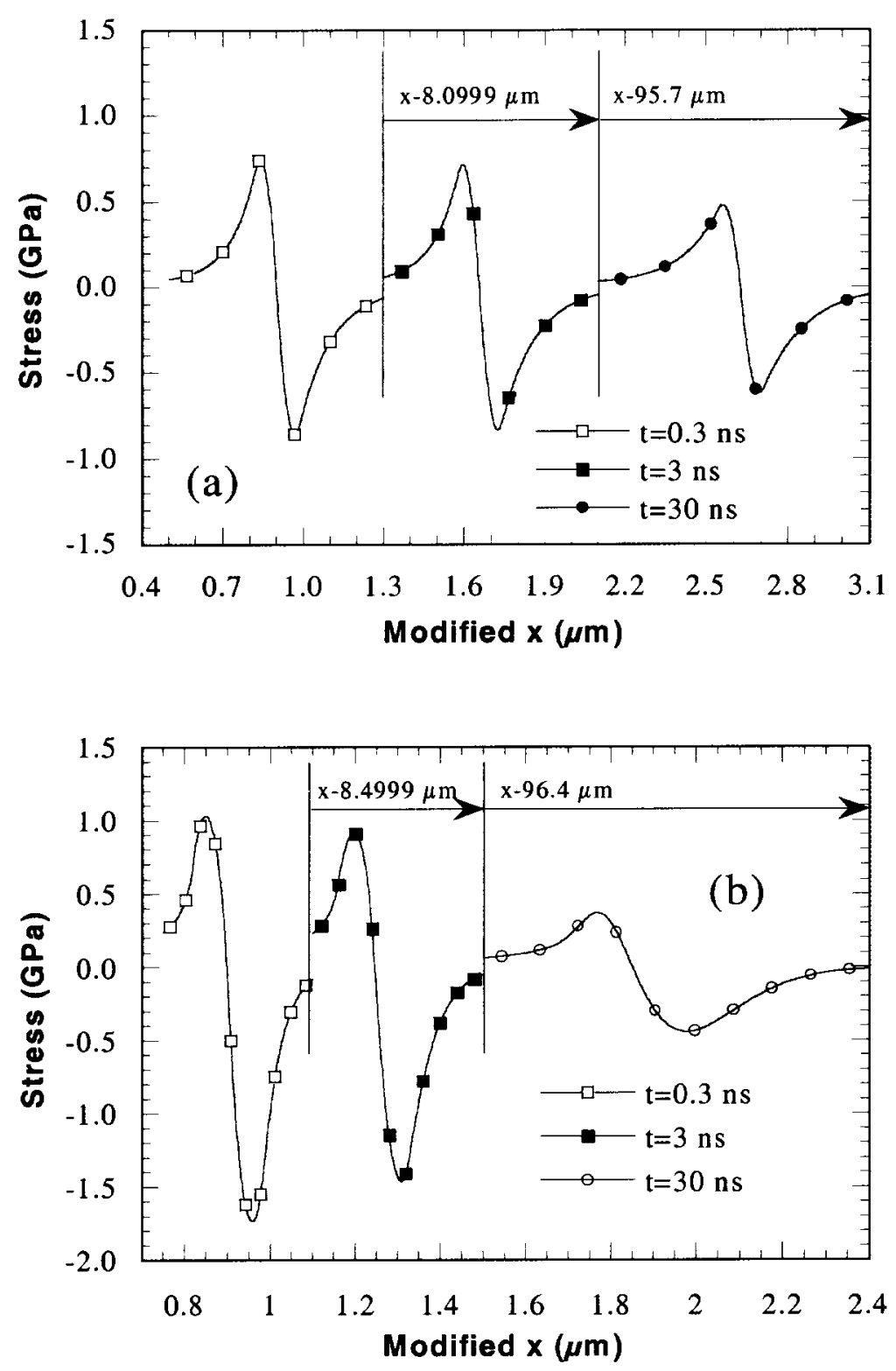

Figure 3. Stress waves in the region far from the surface in gold illuminated with a picosecond laser $(a)$ with the consideration of two-step heat tranfer and $(b)$ without the consideration of two-step heat transfer.

Stress waves in regions far from the surface are shown in Figure $3 a$. For comparison, the stress waves without considering two-step heat transfer are shown in Figure $3 b$. Figure $3 a$ shows that the amplitude of the stress wave at $30 \mathrm{~ns}$ is about $60 \%$ of that at $0.3 \mathrm{~ns}$. This is due to the coupling between the strain rate and the 
lattice temperature. When two-step heat transfer is not considered, the coupling between the strain rate and the lattice temperature is stronger since the faster displacement variation causes stronger attenuation of the stress wave. Figure $3 b$ shows that the tensile stress at $30 \mathrm{~ns}$ is about $37 \%$ of that at $0.3 \mathrm{~ns}$ and the compressive stress is only $26 \%$ of that at $0.3 \mathrm{~ns}$ when two-step heat transfer is not considered. The duration of the stress wave is extended; whereas when two-step heat transfer is considered, the duration of the stress wave remains almost the same during the propagation of the stress wave.

\section{Gold Irradiated with a Femtosecond Pulsed Laser}

In this case, a gold target is irradiated with a femtosecond pulsed laser with a wavelength of $0.8 \mu \mathrm{m}$, a 200 -fs base pulse width, and a peak intensity of $3.061 \times 10^{15} \mathrm{~W} / \mathrm{m}^{2}$ at $100 \mathrm{fs}$.

The surface temperature increase and the displacement are shown in Figures $4 a, b$; the results without considering two-step heat transfer are shown in Figure $4 c$. It is seen from Figure $4 a$ that at around $100 \mathrm{fs}$, the electron gas reaches a peak temperature of about $2.8 \times 10^{5} \mathrm{~K}$. On the other hand, the increase of the lattice temperature is much slower and reaches a much lower peak value of about $1000 \mathrm{~K}$ at $10 \mathrm{ps}$. Unlike the surface displacement induced by picosecond laser heating, which reaches its peak value when the temperature increase stops, the displacement induced by femtosecond laser heating has a significant delay relative to laser heating and the temperature increase. This is because it takes time for the thermal expansion in the laser-heated volume to reach the surface, and this time is significant compared with a femtosecond pulse. Comparing Figures $4 a, c$, a large difference is noticed between the lattice temperatures with and without considering two-step heat transfer. When two-step heat transfer is not considered, the lattice temperature is overevaluated by a factor of 7 and changes with time rapidly. Consequently, the lattice displacement also varies rapidly with time. These phenomena are due to the same reason as explained in picosecond laser heating but are much more pronounced since the heating time here is shorter than the coupling time between electrons and the lattice.

The development of the stress wave during laser heating is shown in Figure 5. It is seen from Figure $5 a$ that the compressive stress is first developed and reaches a peak value at $3.2 \mathrm{ps}$ and then reduces to a constant value. A tensile stress follows the compressive stress and gradually increases to a constant value. Comparing Figure $5 b$ with Figure $5 a$, it is found that the stress is overpredicted without considering two-step heat transfer, especially the tensile stress, which is over 10 times higher than that considering two-step heat transfer. Also, when two-step heat transfer is not considered, the stress wave is completely developed within $10 \mathrm{ps}$. On the other hand, it takes $40 \mathrm{ps}$ when two-step heat transfer is considered.

Propagation and attenuation of the stress waves are shown in Figure $6 a$. It is seen that at $30 \mathrm{~ns}$, the tensile stress is about $53 \%$ of that at $0.3 \mathrm{~ns}$, while the compressive stress is less attenuated, about $65 \%$ of that at $0.3 \mathrm{~ns}$. Comparing Figure $6 b$ with Figure $6 a$, it is clear that without considering two-step heat transfer, the stress 

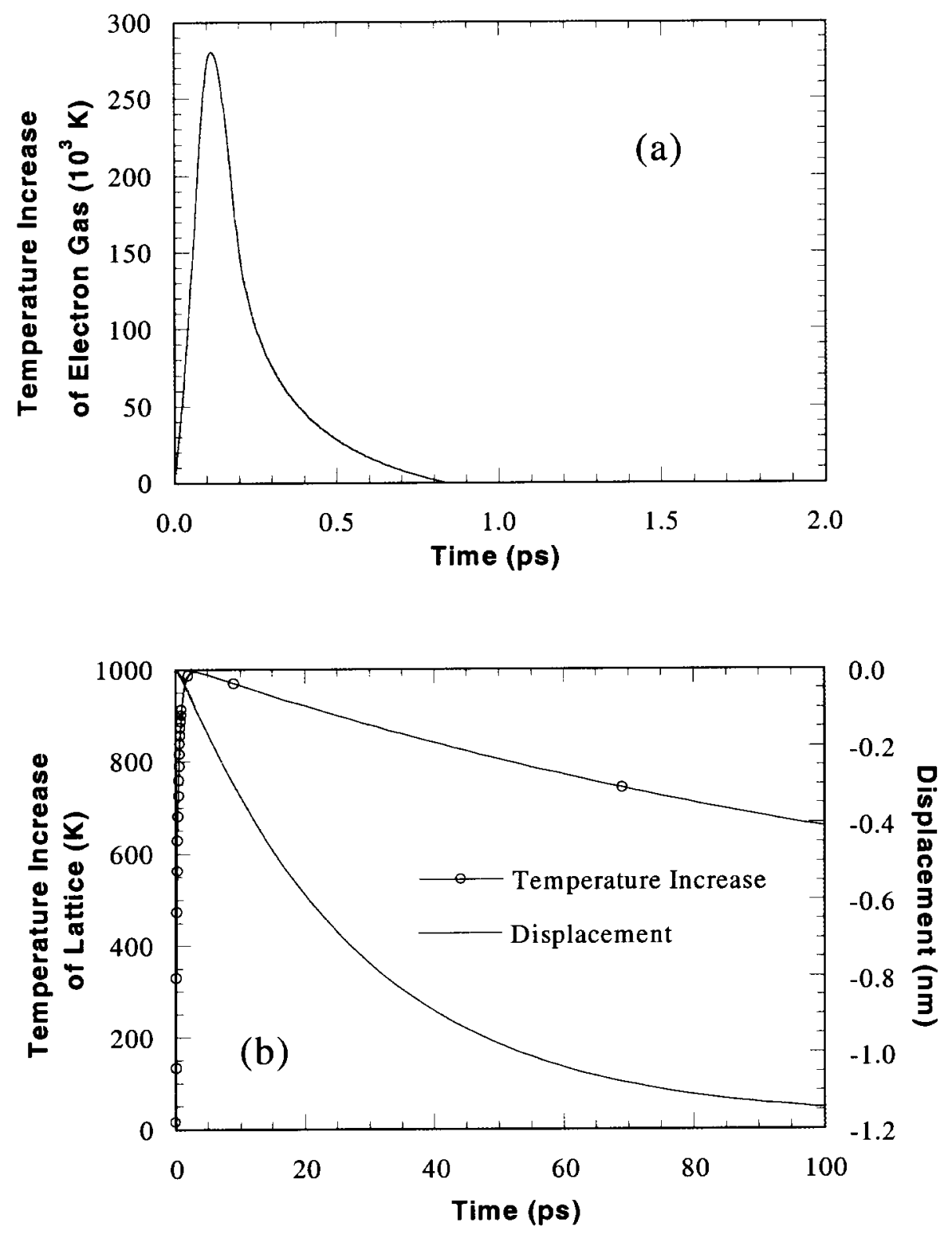

Figure 4. Temperature increase and displacement at the surface of gold illuminated $(a)$ with a femtosecond laser and $(b)$ with the consideration of two-step heat transfer and $(c)$ without the consideration of two-step heat transfer. 


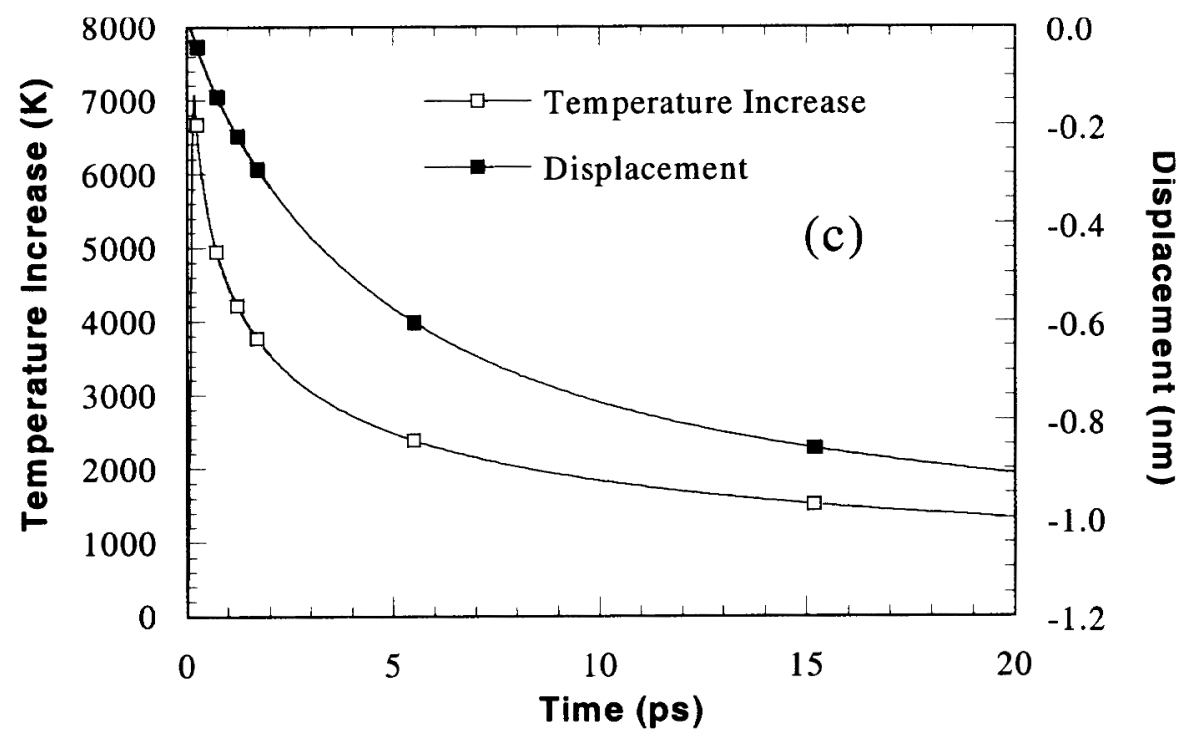

Figure 4. (continued).

wave is attenuated more rapidly and its duration is extended longer. These phenomena are due to the same reason as explained previously in the case of picosecond laser heating.

Comparing the stress wave induced by picosecond and femtosecond laser heating, although the surface temperature increases are almost the same, a shorter pulsed laser induces a stronger stress wave and stronger stress attenuation. Also, it is not difficult to conclude that two-step heat transfer has a larger effect for shorter laser pulses.

Although these calculations are conducted for gold, the solutions developed in this work can be applied for other metals since two-step heat transfer is a common phenomenon in metals.

\section{CONCLUSIONS}

In this work, an analytical solution for thermoelastic waves in a metal induced by a pulsed laser was derived. Two-step heat transfer and coupling between the strain rate and the lattice temperature were considered. Calculations were conducted to investigate the temperature increase and the evolution and attenuation of the stress wave induced by a pulsed laser. Comparing femtosecond laser heating with picosecond laser heating, it was found that the femtosecond laser caused a stronger stress wave and stronger wave attenuation than the picosecond laser did. The displacement at the surface in femtosecond laser heating did not occur simultaneously with the temperature increase but had a substantial delay. It was also shown that, even for a laser pulse longer than the coupling time between electrons and the lattice as in the 

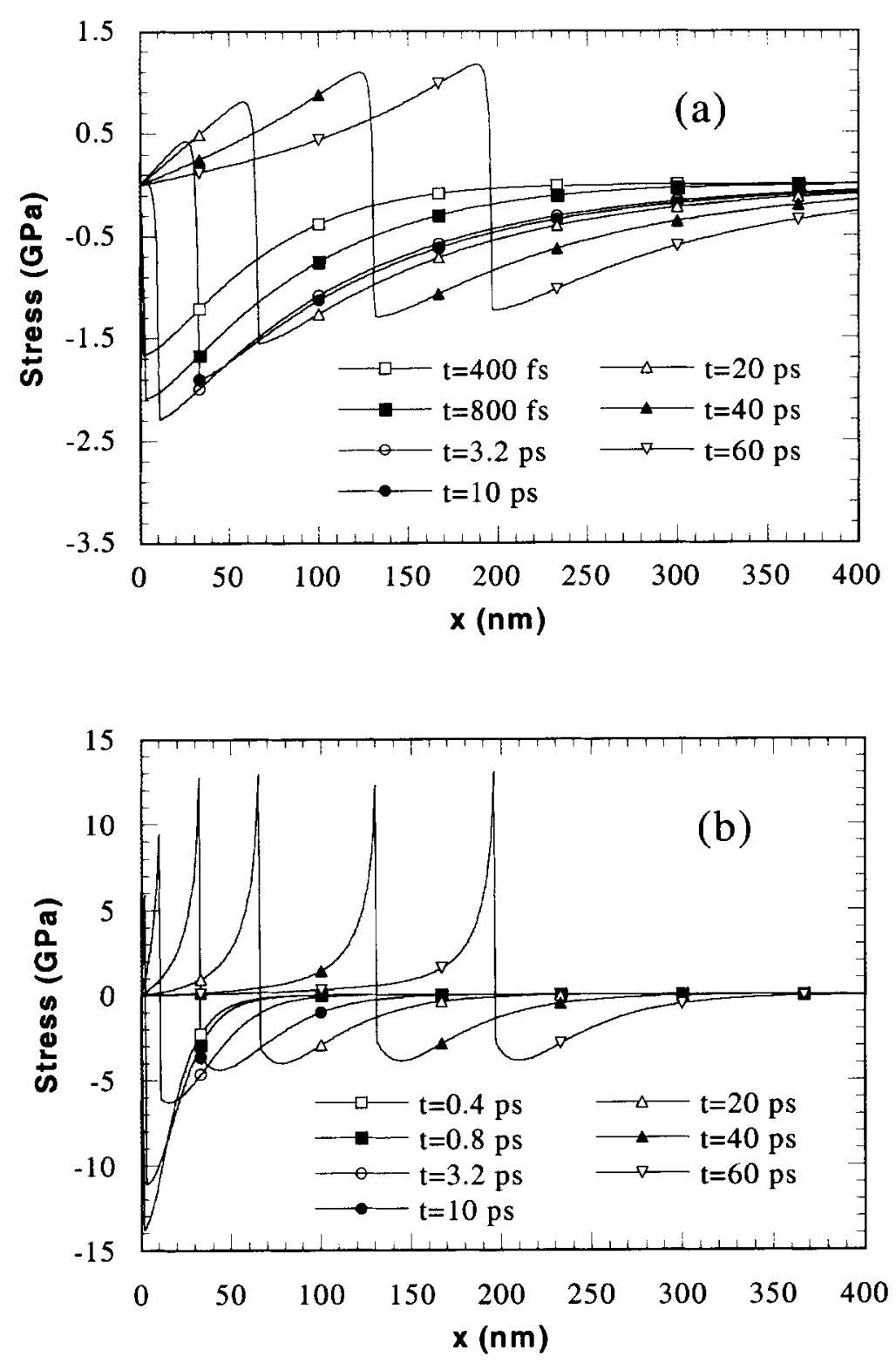

Figure 5. Development of stress waves in gold illuminated with a femtosecond laser $(a)$ with the consideration of two-step heat transfer and $(b)$ without the consideration of two-step heat transfer. 

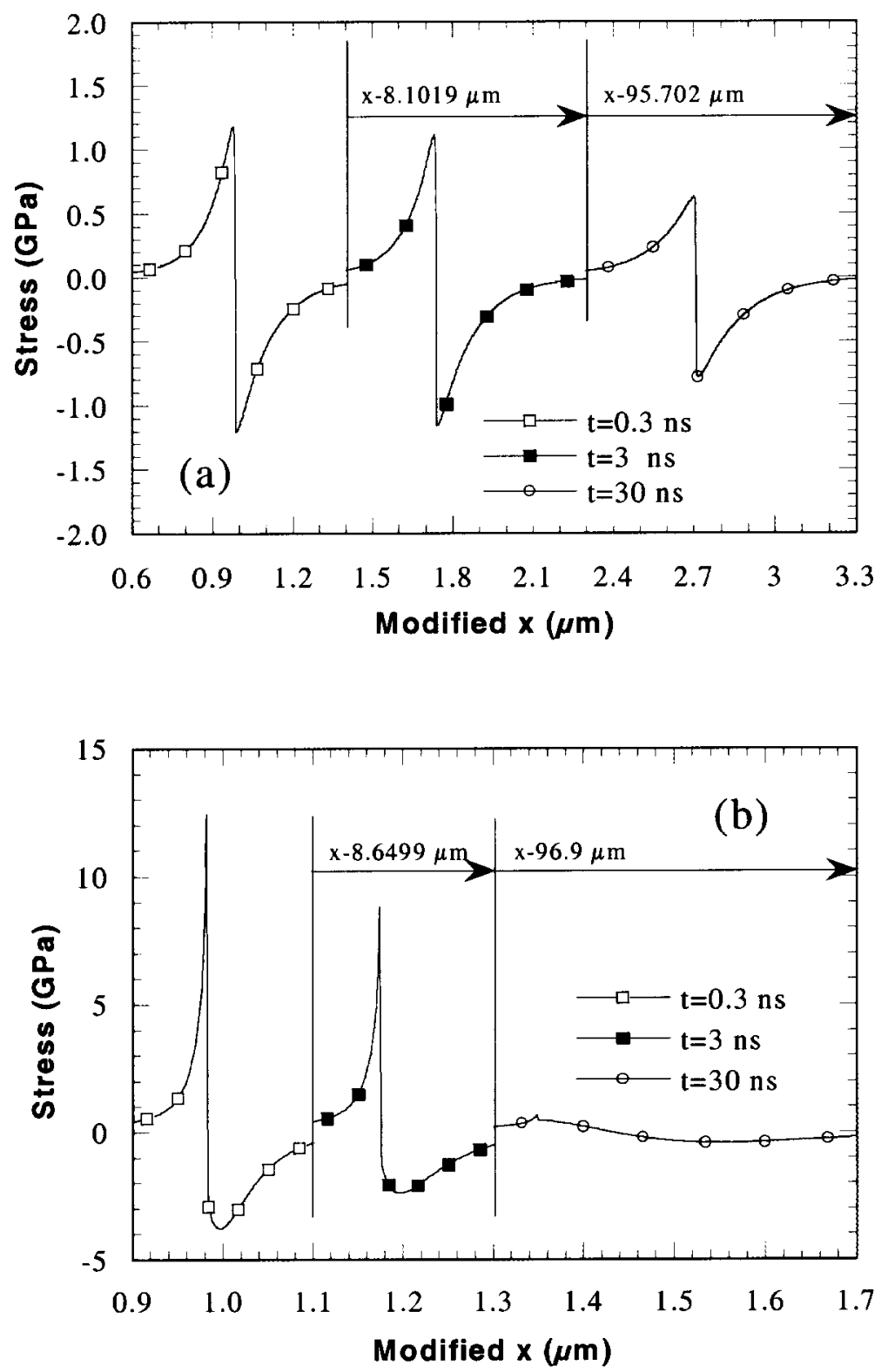

Figure 6. Stress waves in regions far from the surface in gold illuminated with a femtosecond laser $(a)$ with the consideration of the stress wave and $(b)$ without the consideration of the stress wave. 
case of picosecond laser heating, the two-step heat transfer model should still be applied to better predict the temperature and the thermal stress development. Neglecting two-step heat transfer would greatly overpredict the lattice temperature increase, the stress wave, and the coupling between the lattice temperature and the strain rate.

\section{REFERENCES}

1. P. P. Pronko, S. K. Dutta, J. Squier, J. V. Rudd, D. Du, and G. Mourou, Machining of Sub-Micron Holes Using a Femtosecond Laser at 800 nm, Opt. Comm., vol. 114, pp. 106-110, 1995.

2. J. Ihlemann, A. Scholl, H. Schmidt, and B. Wolff-Rottke, Nanosecond and Femtosecond ExcimerLaser Ablation of Oxide Ceramics, Appl. Phys. A, vol. 60, pp. 411-417, 1995.

3. S. Ameer-Beg, W. Perrie, S. Rathbone, J. Wright, W. Weaver, and H. Champoux, Femtosecond Laser Microstructuring of Materials, Appl. Surface Sci., vols. 127-129, pp. 875-880, 1998.

4. K. Kawamura, T. Ogawa, N. Sarukura, M. Hirano, and H. Hosono, Fabrication of Surface Relief Gratings on Transparent Dielectric Materials by Two-Beam Holographic Method Using Infrared Femtosecond Laser Pulses, Appl. Phys. B, vol. 71, pp. 119-121, 2000.

5. X. Zhu, D. M. Villeneuve, A. Yu. Naumov, S. Nikumb, and P. B. Corkum, Experimental Study of Drilling Sub- $10 \mu \mathrm{m}$ Holes in Thin Metal Foils with Femtosecond Laser Pulses, Appl. Surface Sci., vol. 152, pp. 138-148, 1999.

6. B. Bonello, B. Perrin, E. Romatet, and J. C. Jeannet, Application of the Picosecond Ultrasonic Technique to the Study of Elastic and Time-Resolved Thermal Properties of Materials, Ultrasonics, vol. 35, pp. 223-231, 1997.

7. C. J. Morath, H. J. Maris, J. J. Cuomo, D. L. Pappas, A. Grill, V. V. Patel, J. P. Doyle, and K. L. Saenger, Picosecond Optical Studies of Amorphous Diamond and Diamondlike Carbon: Thermal Conductivity and Longitudinal Sound Velocity, J. Appl. Phys., vol. 76, pp. 2636-2640, 1994.

8. J. L. Hostetler, A. N. Smith, and P. M. Norris, Simultaneous Measurement of Thermophysical and Mechanical Properties of Thin Films, Int. J. Thermophys., vol. 19, pp. 569-577, 1998.

9. W. S. Capinski, M. Cardona, D. S. Katzer, H. J. Maris, K. Ploog, and T. Ruf, Thermal Conductivity of GaAs/AlAs, Phys. B, vols. 263-264, pp. 530-532, 1999.

10. C. Bai and A. S. Lavine, On Hyperbolic Heat Conduction and the Second Law of Thermodynamics, J. Heat Transfer, vol. 117, pp. 256-263, 1995.

11. D. W. Tang and N. Araki, The Wave Characteristics of Thermal Conduction in Metallic Films Irradiated by Ultra-Short Laser Pulses, J. Phys. D: Appl. Phys., vol. 29, pp. 2527-2533, 1996.

12. D. W. Tang and N. Araki, Wavy, Wavelike, Diffusive Thermal Response of Finite Rigid Slabs to High-Speed Heating of Laser-Pulses, Int. J. Heat Mass Transfer, vol. 42, pp. 855-860, 1999.

13. X. Wang and X. Xu, Thermoelastic Wave Induced by Pulsed Laser Heating, Appl. Phys. A, 2001 (in press).

14. S. I. Anisimov, B. L. Kapeliovich, and T. L. Perel'man, Electron Emission from Metal Surfaces Exposed to Ultra-Short Laser Pulses, Soviet Phys. JETP, vol. 39, pp. 375-377, 1974.

15. T. Q. Qiu and C. L. Tien, Heat Transfer Mechanisms during Short-Pulse Laser Heating of Metals, J. Heat Transfer, vol. 115, pp. 835-841, 1993.

16. D. Y. Tzou, A Unified Field Approach for Heat Conduction From Macro- to Microscales, J. Heat Transfer, vol. 117, pp. 8-16, 1995.

17. D. Y. Tzou, Macro- to Microscale Heat Transfer-The Lagging Behavior, Chs. 5 and 9, Taylor and Francis, Washington, DC, 1996.

18. B. A. Boley and I. S. Tolins, Transient Coupled Thermoelastic Boundary Value Problems in the HalfSpace, J. Appl. Mech., vol. 29, pp. 637-646, 1962.

19. R. M. White, Generation of Elastic Waves by Transient Surface Heating, J. Appl. Phys., vol. 34, pp. 3559-3567, 1963.

20. H. W. Lord and Y. Shulman, A Generalized Dynamical Theory of Thermoelasticity, J. Mech. Phys. Solids, vol. 15, pp. 299-309, 1967. 
21. J. C. Bushnell and D. J. McCloskey, Thermoelastic Stress Production in Solids, J. Appl. Phys., vol. 39, pp. 5541-5546, 1968.

22. T. T. Kao, On Thermally Induced Non-Fourier Stress Waves in a Semi-Infinite Medium, AIAA J., vol. 14, pp. 818-820, 1976.

23. L. P. Welsh, J. A. Tuchman, and I. P. Herman, The Importance of Thermal Stresses and Strains Induced in Laser Processing with Focused Gaussian Beams, J. Appl. Phys., vol. 64, pp. 6274-6286, 1988.

24. F. A. McDonald, On the Precursor in Laser-Generated Ultrasound Waveforms in Metals, Appl. Phys. Lett., vol. 56, pp. 230-232, 1990.

25. J. Mozina and M. Dovc, One-Dimensional Model of Optically Induced Thermoelastic Waves, Modern Phys. Lett. B, vol. 8, pp. 1791-1801, 1994.

26. A. Galka and R. Wojnar, One-Dimensional Dynamic Thermal Stresses Generated in an Elastic HalfSpace by Laser Pulses, J. Thermal Stresses, vol. 18, pp. 113-140, 1995.

27. D. S. Chandrasekharaiah and K. S. Srinath, Thermoelastic Plane Waves without Energy Dissipation in a Half-Space due to Time-Dependent Heating of the Boundary, J. Thermal Stresses, vol. 20, pp. 659-676, 1997.

28. F. Enguehard and L. Bertrand, Effects of Optical Penetration and Laser Pulse Duration on Laser Generated Longitudinal Acoustic Waves, J. Appl. Phys., vol. 82, pp. 1532-1538, 1997.

29. D. W. Tang and N. Araki, Non-Fourier Heat Conduction in a Finite Medium under Periodic Surface Thermal Disturbance-II. Another Form of Solution, Inter. J. Heat Mass Transfer, vol. 39, pp. 33053308, 1996.

30. R. B. Hetnarski and J. Ignaczak, Generalized Thermoelasticity, J. Thermal Stresses, vol. 22, pp. 451476, 1999.

31. D. S. Chandrasekharaiah, Hyperbolic Thermoelasticity: A Review of Recent Literature, Appl. Mech. Rev., vol. 51, pp. 705-729, 1998.

32. E. G. Gamaly, A. V. Rode, and B. Luther-Davies, Ultrafast Ablation with High-Pulse-Rate Lasers. Part I: Theoretical Considerations, J. Appl. Phys., vol. 85, pp. 4213-4221, 1999.

33. M. D. Feit, A. M. Rubenchik, B.-M. Kim, L. B. da Silva, and M. D. Perry, Physical Characterization of Ultrashort Laser Pulse Drilling of Biological Tissue, Appl. Surface Sci., vols. 127-129, pp. 869-871, 1998.

34. E. N. Sobol, Phase Transformations and Ablation in Laser-Treated Solids, Ch. 1, John Wiley \& Sons, New York, 1995.

35. C. Thomsen, H. T. Grahn, H. J. Maris, and J. Tauc, Surface Generation and Detection of Phonons by Picosecond Light Pulses, Phys. Rev. B, vol. 34, pp. 4129-4138, 1986.

36. B. Bonello, B. Perrin, E. Romatet, and J. C. Jeannet, Application of the Picosecond Ultrasonic Technique to the Study of Elastic and Time-Resolved Thermal Properties of Materials, Ultrasonics, vol. 35 , pp. 223-231, 1997.

37. S. D. Brorson, J. G. Fujimoto, and E. P. Ippen, Femtosecond Electronic Heat-Transfer Dynamics in Thin Gold Films, Phys. Rev. Lett., vol. 59, pp. 1962-1965, 1987.

38. C. Kittel, Introduction to Solid State Physics, Ch. 6, John Wiley \& Sons, New York, 1976.

39. W. S. Fann, R. Storz, H. W. K. Tom, and J. Bokor, Direct Measurement of Nonequlibrium ElectronEnergy Distributions in Subpicosecond Laser-Heated Gold Films, Phys. Rev. Lett., vol. 68, pp. 2834 2837, 1992.

40. F. P. Incropera and D. P. DeWitt, Fundamentals of Heat and Mass Transfer, Appendix A, John Wiley \& Sons, New York, 1996.

41. D. R. Lide, CRC Handbook of Chemistry and Physics: a Ready-Reference Book of Chemical and Physical Data, Section 12, CRC Press, Boca Raton, FL, 1994. 